\title{
Avaliação da qualidade nutricional em espécies de pescado mais produzidas no Estado da Bahia
}

\author{
Quality evaluate nutritional the fisches more consumed in State of Bahia - Brazil
}

\author{
Graciele de Queiroz ANDRADE ${ }^{1}$, Eliete da Silva BISPO ${ }^{1 *}$, Janice Izabel DRUZIAN ${ }^{1}$
}

\begin{abstract}
Resumo
Diversos fatores podem contribuir para a variabilidade na composição das diferentes espécies de pescado. Este trabalho teve como objetivo, identificar as espécies de pescado mais produzidas no Estado da Bahia, avaliar seu valor nutricional e realizar um levantamento das empresas beneficiadoras de pescado do Estado. Um questionário foi aplicado às beneficiadoras de pescado para a caracterização das empresas. Segundo a Bahia Pesca (2005), as espécies mais produzidas são: o camarão-rosa (Penaeus brasiliensis), representando 21,8\% da produção estadual; a sardinha (Opisthonema oglinum), 10,2\%; os vermelhos com as espécies guaiúba (Ocyurus chrysurus) e o ariacó (Lutjanus synagris), 6,6\%; seguidos pela tainha (Mugil spp.), 4,1\%. De acordo com o valor nutricional das espécies analisadas, a guaiúba foi considerada melhor para o consumo, por possuir menor teor de lipídios totais $(0,82 \%)$ e gordura saturada (28,74\%). A produção de $43 \%$ das beneficiadoras de pescado do Estado da Bahia está entre 10 e 50 ton/mês e a principal dificuldade apresentada foi a falta de investimento do governo no setor.

Palavras-chave: composição química; nutrientes em pescado; ácidos graxos; beneficiamento.
\end{abstract}

\begin{abstract}
Several factors can contribute to the variability in the composition of the different species of fish. The objective of this work was to identify the most frequently consumed fish species in the State of Bahia to evaluate their nutritional value and to compile a list of fish processing companies in the State. A questionnaire was applied to the companies to characterize them. According to Bahia Pesca (2005), the most frequently consumed species are the shrimp-rose (Penaeus brasiliensis), accounting for $21.8 \%$ of the state production, the sardine (Opisthonema oglinum), $10.2 \%$, and the reds, especially guaiúba (Ocyurus chrysurus) and ariacó (Lutjanus synagris), 6.6\%, followed by the mullet (Mugil spp.), 4.1\%, respectively. Considering the nutritional value of the analyzed fish, the guaiúba was considered the best for consumption due to its smaller content of total lipids ( $0.82 \%)$ and saturated fat $(28.74 \%)$. The production of $43 \%$ of the companies of the State of Bahia is around 10 to 50 ton/month, and the major difficulty found was poor investment in the sector by the government.

Keywords: chemical composition; fish nutrients; fatty acids; benefit.
\end{abstract}

\section{Introdução}

Em várias partes do mundo, a exploração de pescado voltado para a produção de alimentos tem uma importância considerável. Na Bahia, devido às suas características geográficas de extensa zona costeira, os peixes, camarões e moluscos são extremamente apreciados pela população, através da elaboração de pratos típicos da culinária baiana e representando subsistência da população do Recôncavo Baiano.

A produção pesqueira do Estado da Bahia foi estimada em 45.631,00 toneladas em 2005, sendo a maior da região Nordeste (BAHIA PESCA, 2005). A produção da Bahia representa aproximadamente $11,92 \%$ da produção brasileira (382.652,5 toneladas) e $0,03 \%$ da produção mundial de peixes. Segundo dados da Organização das Nações Unidas para Agricultura e Alimentação, em 2005, a produção de peixe no mundo foi de 142 milhões de toneladas, com um aumento de mais de 1 milhão de toneladas, quando comparada à de 2004 . Desta, cerca de 103 milhões de toneladas foram de origem marinha (FAO, 2007; IBAMA, 2006). Os mercados de peixes frescos, congelados, enlatados e secos são os principais destinos do pescado, representando aproximadamente $76 \%$ da produção mundial (FAO, 2007).

Diversos trabalhos têm destacado a grande utilidade do pescado como fonte alimentar, graças ao seu valor nutritivo, fácil digestibilidade, diversidade de sabores e composição equilibrada. A composição do pescado e consequentemente o seu valor nutritivo variam em função de numerosos fatores: espécie a que pertence, idade, meio em que vive, tipo de alimentação, época de captura, peso, entre outros (ALMEIDA;FRANCO, 2006; GOKÇE, 2004; LUZIA et al., 2003; OGATA et al., 2004; OKADA; MORRISSEY, 2007; RECKS; SEABORN, 2007; ZLATANOS; LASKARIDIS, 2007).

Em geral, a composição do pescado varia em umidade numa proporção de 64 a 90\%; em proteína, de 8 a 23\%; em gordura, de

Recebido para publicação em 22/2/2008

Aceito para publicação em 3/1/2009 (003032)

Departamento de Análises Bromatológicas, Faculdade de Farmácia, Universidade Federal da Bahia, Av. Barão de Jeremoabo s/n, CEP 40171-970 Ondina, Salvador - BA, Brasil, E-mail:elibispo@ufba.br

${ }^{*}$ A quem a correspondência deve ser enviada 
0,5 a 25\%; em resíduos minerais, de 1 a 2\%; e em carboidratos, menos que 1\% (HART; FISHER, 1971; STANSBY, 1973). O pescado contém elevado nível de proteínas de alto valor biológico e alta digestibilidade (BADOLATO et al., 1994a). Ackman (1989) dividiu os peixes em quatro categorias quanto ao seu teor de lipídios: magros (menor que $2 \%$ de gordura); baixo teor de gordura (2-4\% de gordura); semigordo (4-8\% de gordura); e altamente gordo (maior que $8 \%$ de gordura).

A investigação da composição química, particularmente com relação à composição de ácidos graxos no conteúdo lipídico do pescado, vem despertando grande interesse pela comunidade científica mundial, pois está relacionada diretamente à saúde humana. Dentre estes ácidos graxos, os pertencentes à família ômega-3, como o ácido eicosapentaenóico (EPA) e o docosahesaenóico (DHA) têm recebido maior atenção por reduzirem fatores de risco associados a doenças cardiovasculares, hipertensão, inflamações em geral, asma, artrite, psoríase e de vários tipos de câncer (CASTRO-GONZALEZ, 2002; LIMA et al., 2004; MARTIN et al., 2006; VON SCHACKY, 2007; WANG et al., 2006). Por outro lado, os ácidos graxos saturados aumentam o nível de colesterol sanguíneo, por reduzir a atividade do receptor LDL-colesterol, reduzir o espaço livre de LDL na corrente sanguínea e bloquear a enzima $\Delta-6$ dessaturase e, consequentemente, na produção de ácidos graxos importantes como EPA e DHA (SCHMIDT, 2000).

Levando-se em consideração as diferenças encontradas para um mesmo produto entre as várias tabelas de composição de alimentos, a caracterização do produto regional é importante não só para a elaboração de tabelas de balanço nutricional ou para o cálculo de ingestão de nutrientes como também para viabilizar um melhor aproveitamento deste pescado e servir de apoio à criação de novas perspectivas industriais, com agregação de valor econômico ao setor e consequentes respostas sociais.

Dessa forma, esse trabalho teve o objetivo de identificar as espécies de pescado mais produzidas do Estado da Bahia, avaliar o seu valor nutricional, além de realizar um levantamento das empresas beneficiadoras de pescado no Estado.

\section{Material e métodos}

\subsection{Coleta e armazenamento do pescado}

Foram escolhidas, para avaliação nutricional, as cinco espécies de pescado mais produzidas no Estado da Bahia, segundo dados publicados pela Bahia Pesca (BAHIA PESCA, 2005): a sardinha (Opisthonema oglinum); a tainha (Mugil spp.); o camarão (Penaeus brasiliensis); e as espécies de vermelhos guaiúba (Ocyurus chrysurus) e o ariacó (Lutjanus synagris).

As espécies foram coletas mensalmente no Mercado Municipal de Frutos do Mar (Salvador-BA), durante os meses de março, abril, maio e junho no ano de 2004, sendo adquirido um total de $1 \mathrm{~kg}$ a cada coleta. Não se levaram em consideração sexo, idade e estágio de maturação. Este procedimento foi realizado para que os dados obtidos correspondam a uma média composicional confiável que represente a população explorada e não apenas alguns organismos.
As amostras foram adquiridas frescas, imediatamente acondicionadas entre camadas de gelo em caixas de isopor e transportadas para o Laboratório de Pescado e Cromatografia Aplicada (LAPESCA) da Faculdade de Farmácia-UFBA. No laboratório, os músculos dorsais foram extraídos manualmente (peixes ainda frescos), triturados em processador e armazenados $\mathrm{a}-18{ }^{\circ} \mathrm{C}$ em diferentes porções até o momento das análises.

\subsection{Determinações físicas de peso do pescado}

$\mathrm{O}$ peso total e individual das espécies foram obtidos por pesagem em balança "FILIZOLA", com o máximo de $20 \mathrm{Kg}$ e divisão de $20 \mathrm{~g}$, os registros foram feitos em décimo de gramas.

\subsection{Determinação da composição centesimal do músculo}

A umidade foi determinada de acordo com método da AOAC (1997) por perda de peso após o aquecimento em estufa a $105^{\circ} \mathrm{C}$ até peso constante. Os lipídios totais foram determinados pelo método Bligh e Dyer (1989). O conteúdo de proteína foi determinado segundo método descrito na AOAC (1997). A determinação dos resíduos minerais foi realizada segundo método descrito na AOAC (1997). Os minerais, teores de cálcio, ferro e sódio foram analisados segundo método descrito por Vogel (1992).

A fração "Nifext" (nitrogen free extract) foi determinada através das somas das demais determinações subtraídas de 100. O valor calórico foi determinado através de metodologias oficiais Krausse; Mahan; Escott-Stump (1998).

\subsection{Determinação dos ácidos graxos saturados}

\section{Derivação da fração lipídica}

Para a determinação da composição de ácidos graxos saturados da fração lipídica, foi realizada a esterificação dos ácidos graxos dos lipídios totais, segundo Joseph e Ackman (1992). Uma alíquota dos lipídios totais (aproximadamente $25 \mathrm{mg}$ ) foi submetida à reação de saponificação com $\mathrm{NaOH} 0,5 \mathrm{~mol} / \mathrm{L}$ em metanol, seguida de metilação com catalisador $\mathrm{BF}_{3}(12 \%$ em metanol). Todas as etapas do processo foram realizadas sob atmosfera de $\mathrm{N}_{2}$ gasoso.

\section{Análise da composição de ácidos graxos}

A separação dos metil ésteres de ácidos graxos saturados foi realizada em cromatógrafo a gás (Varian 3800) equipado com detector de ionização de chama (CG-DIC) e coluna capilar de sílica fundida ELITE-WAX (30 $\mathrm{m} \times 0,32 \mathrm{~mm} \times 0,25 \mu \mathrm{m})$. Os parâmetros de análises foram: temperatura do injetor $250{ }^{\circ} \mathrm{C}$; temperatura do detector $280{ }^{\circ} \mathrm{C}$; temperatura da coluna programada a $150{ }^{\circ} \mathrm{C}$ por 16 minutos e aumentando $2{ }^{\circ} \mathrm{C}$ por minuto até $180^{\circ} \mathrm{C}$; permanecendo nessa temperatura por 25 minutos e aumentando $5^{\circ} \mathrm{C}$ até $210^{\circ} \mathrm{C}$; e permanecendo nessa temperatura por 25 minutos. O gás hélio foi utilizado como gás de arraste a 1,3 $\mathrm{mL} / \mathrm{minuto}$, com velocidade linear $38 \mathrm{~cm} /$ segundo e filtro de oxigênio acoplado à linha de gás. Foi utilizado nitrogênio como gás make up a $30 \mathrm{~mL} /$ minuto; fluxo do gás hidrogênio a $30 \mathrm{~mL} /$ minuto; ar sintético a $300 \mathrm{~mL} /$ minuto; sendo que a 
técnica de injeção foi split na razão 1:50. As injeções foram realizadas em duplicatas para cada extração (com total de quatro injeções para cada amostra) e o volume de injeção foi de $1 \mu \mathrm{L}$.

A identificação dos ácidos graxos foi realizada por comparação dos tempos de retenção dos picos das amostras com o tempo de retenção dos ésteres metílicos de ácidos graxos de padrão mix (189-19, Sigma, EUA). Como parâmetro decisivo à identificação dos ácidos graxos, foi utilizado um espectrômetro de massas (Clarus 500) acoplado a um cromatógrafo a gás (Pelkin Elmer), por comparação a dados espectrais da biblioteca NIST (do equipamento) e padrão mix com os das amostras, nas mesmas condições cromatográficas operacionais usadas no CG-DIC e espectro entre 45-600 m/z (IE, 70ev).

A quantificação dos ácidos graxos foi realizada pelo método de normalização através das áreas dos picos e os resultados de ácidos graxos foram expressos em percentagem relativa de área (\%).

\subsection{Aplicação do questionário às empresas beneficiadoras de pescado}

Um questionário foi elaborado, buscando reunir detalhes para identificação das empresas (Quadro 1). As principais indústrias beneficiadoras de pescado foram identificadas através de um cadastro realizado pela Bahia Pesca. Foi aplicado o questionário para um total de 9 (nove) indústrias representando $45 \%$ do total das empresas beneficiadoras cadastradas do Estado da Bahia.

\section{Resultados e discussão}

As espécies de pescado apresentaram os valores de peso médio compreendidos entre 40,00 e 532,75 g, sendo o menor peso apresentado pelo camarão e o maior pela guaiúba (Tabela 1).

A Tabela 1 apresenta os valores de composição centesimal das espécies analisadas. $\mathrm{O}$ teor de proteínas apresentou uma pequena variação entre as espécies, com valores entre 19,48 e 20,26\% para guaiúba (Ocyurus chrysurus) e tainha (Mugil spp.), respectivamente.

Os teores de lipídios totais foram os que demonstraram a maior variação entre as espécies analisadas, com base na matéria úmida o menor teor foi encontrado para a guaiúba $(0,82 \%)$ e o maior foi obtido para a tainha (4,39\%). A sardinha (Sardinella brasiliensi) analisada por Pessatti (2000) apresentou teor lipídico de $7,7 \%$, valor foi superior ao encontrado no presente trabalho para a sardinha (Opisthonema oglinum) (2,35\%). De acordo com a classificação de Ackman (1989), o ariacó e a guaiúba foram considerados peixes "magros", a sardinha e o camarão classificados como "baixo teor de gordura" e a tainha como "semigordo".

Os teores de carboidratos encontrados estão de acordo com os dados em literatura para esse macronutriente, com valores similares aos resultados obtidos por Nunes (1976) e Ogawa; Perdigão (1979).
Nome da empresa:

Tel:

Endereço:

1. Qual o número de empregados da sua empresa?
a) $<20$
b) 20 a 50
c) 50 a 100
d) $>100$

2. Qual a produção mensal por espécie?

a) $<10$ toneladas

b) 10 a 50 toneladas

c) 50 a 100 toneladas

d) $>100$ toneladas

3. Qual a capacidade máxima instalada de produção da empresa?

a) $500 \mathrm{~m}^{2}$

b) $600 \mathrm{~m}^{2}$ a $800 \mathrm{~m}^{2}$

c) $800 \mathrm{~m}^{2}$ a $1000 \mathrm{~m}^{2}$

d) $>1000 \mathrm{~m}^{2}$

4. Quais as espécies de pescado utilizadas como matéria-prima?

a) tainha

b) sardinha

c) agulha

d) camarão

e) outras espécies(quais?)

5. As espécies de pescado são capturadas ou produzidas em cativeiros?
a) cativeiro
b) capturada

6. Quais os produtos finais da empresa?

a) pescado congelado inteiro

b) pescado filetado congelado

c) produto industrializado (quais?)

7. Qual a origem do pescado da empresa?
a) da região
b) outra região
c) se outra, qual?

8. Qual o principal mercado do destino da produção da empresa?
a) Nordeste
b) Sul
c) Sudeste
d) internacional

9. Quais as dificuldades enfrentadas no beneficiamento do pescado?

a) sazonalidade da matéria-prima

b) conservação do pescado até a indústria

c) novos recursos pesqueiros

d) falta de um padrão do tamanho da matéria-prima

e) mão de obra qualificada

f) falta de investimento do governo

10. Qual o volume total do resíduo?

a) $<1 / 2$ tonelada mensal

b) 1 tonelada mensal

c) $>1$ tonelada mensal

11. Qual o destino do resíduo?
a) aterro
b) rio
c) $\mathrm{mar}$
d) outros (quais?)

12. A empresa tem interesse no aproveitamento do resíduo?

$\begin{array}{ll}\text { a) } \operatorname{sim} & \text { b) não }\end{array}$

13. Se sim qual o produto de interesse?

a) farinha de pescado

b) extração do óleo

c) outros produtos

14. A empresa já efetuou algum programa de qualidade?

$\begin{array}{lll}\text { a) } \operatorname{sim} & \text { b) não } & \text { c) se sim, qual? }\end{array}$

Quadro 1. Questionário aplicado às empresas de beneficiamento de pescado do Estado. 
O resíduo mineral fixo oscilou em torno de $1 \%$ na matéria úmida, sendo o menor teor $(1,11 \%)$ encontrado para a tainha e o maior $(1,70 \%)$ para a sardinha (Opisthonema oglinum). Resultados semelhantes foram encontrados por Pessatti (2000), que obteve teor de 1,6\% para a Sardinella brasiliensis.

$\mathrm{Na}$ análise dos teores de cálcio, ferro e sódio, o camarão destacou-se como maior fonte de cálcio (76,44 mg.100 g $\left.\mathrm{g}^{-1}\right) \mathrm{e}$ a sardinha como maior teor de sódio (184 mg.100 g-1); foram verificadas quantidades consideráveis desses minerais em todas as espécies analisadas no presente estudo (Tabela 1).

Os resultados referentes à composição de ácidos graxos saturados para as diferentes espécies estudadas podem ser observadas na Tabela 2 .

Os ácidos graxos saturados estão relacionados ao aumento dos níveis de colesterol plasmático, podendo estar relacionados à formação de placas de ateromas no organismo humano (SCHMIDT, 2000).

Nas espécies analisadas no presente estudo, o ácido palmítico (C16:0) apresentou a maior concentração $(15,35$ a 24,02\%), seguido do ácido esteárico (C18:0) (5,04 a 10,90\%). A soma de todos os ácidos graxos saturados identificados variou entre 28,74 e $40,62 \%$ (Tabela 2), sendo o menor valor relativo à guaiúba e o maior à sardinha. Resultados semelhantes foram encontrados por Sanchez-Muniz (1992) na sardinha (Sardina pilchardus), o qual verificou um total de $42,0 \%$ de ácidos graxos saturados, sendo os ácidos palmítico (C16:0) e esteárico (C18:0) os majoritários com 27,6 e 9,6\%, respectivamente. Resultados esses semelhantes aos encontrados neste experimento para o músculo da sardinha bandeira (Ophistonema oglinum).

Comparando os ácidos graxos saturados majoritários da tainha (Mugil spp) estudados por Badolato et al. (1994b) aos encontrados neste experimento, foi verificado que também ocorreu predominância dos ácidos graxos palmítico (21,72\%) e esteárico (10,27\%).

Estabelecendo uma comparação entre as espécies ariacó (Lutjanus synagris) e guaiúba (Ocyurus chrysurus) que pertencem à mesma família Lutjanidea, foi observada a mesma predominância dos ácidos graxos saturados, em concordância com os resultados obtidos por Yusuf et al.,(1993) em Redsnapper (Lutianus spp) e por Viswanathan; Gopakumar (1978) em Waigue Snapper (Lutianus waigiensis).

Caracterizando o perfil lipídico de ácidos graxos de amostras comerciais de camarão-rosa (Penaeus brasiliensis e Penaeus paulensis), Moura et al.,(2002) revelaram a presença de $32,9 \%$ de ácidos graxos saturados, sendo o C16:0 $(18,2 \%)$ e o 18:0 (10,1\%) os mais encontrados nesse grupo.

De acordo com a RDC no 360 de 23 de dezembro de 2003 (BRASIL, 2003), a quantidade de ácidos graxos saturados encontrados nas espécies analisadas é considerada como "não significativa" ou quantidade "zero" (Tabela 3).

A tainha e a guaiúba foram as espécies que apresentaram maior (70 kcal/60 g) e menor (50 kcal/60 g) teor energético, respectivamente. O teor energético apresentou uma relação com o teor de gordura, condizente com o estudo de Rocha et al., (1982).

Tabela 1. Composição centesimal, peso médio e valor calórico do músculo das espécies mais produzidas no Estado da Bahia.

\begin{tabular}{lcccccccrr}
\hline \multicolumn{1}{c}{ Espécie } & $\begin{array}{c}\text { Peso } \\
\text { médio }(\mathrm{g})\end{array}$ & $\begin{array}{c}\text { Umidade } \\
(\%)\end{array}$ & $\begin{array}{c}\text { Lipídio } \\
(\%)\end{array}$ & $\begin{array}{c}\text { Proteína } \\
(\%)\end{array}$ & $\begin{array}{c}\text { Cinzas } \\
(\%)\end{array}$ & $\begin{array}{c}\text { Carboidratos } \\
(\%)\end{array}$ & $\begin{array}{c}\text { Cálcio } \\
\left(\mathrm{mg}^{2} \cdot 100 \mathrm{~g}^{-1}\right)\end{array}$ & $\begin{array}{c}\text { Ferro } \\
\left(\mathrm{mg}^{2} \cdot 100 \mathrm{~g}^{-1}\right)\end{array}$ & $\begin{array}{c}\text { Sódio } \\
\left(\mathrm{mg}^{2} \cdot 100 \mathrm{~g}^{-1}\right)\end{array}$ \\
\hline Sardinha & 63,23 & $75,25 \pm 1,89$ & $2,35 \pm 0,46$ & $20,25 \pm 0,95$ & $1,70 \pm 0,20$ & $0,75 \pm 0,17$ & $25,36 \pm 7,08$ & $0,71 \pm 0,37$ & $184,54 \pm 0,56$ \\
Tainha & 199,33 & $76,45 \pm 1,86$ & $4,39 \pm 3,31$ & $20,26 \pm 1,12$ & $1,11 \pm 0,14$ & $0,79 \pm 0,63$ & $15,42 \pm 0,19$ & $0,58 \pm 0,13$ & $98,07 \pm 0,62$ \\
Camarão & 40,00 & $76,60 \pm 0,02$ & $2,52 \pm 0,40$ & $19,58 \pm 0,61$ & $1,30 \pm 0,14$ & - & $76,44 \pm 1,20$ & $0,20 \pm 0,02$ & $163,20 \pm 0,54$ \\
Ariacó & 423,00 & $77,38 \pm 1,55$ & $1,33 \pm 0,48$ & $19,73 \pm 1,72$ & $1,12 \pm 0,10$ & $0,46 \pm 0,47$ & $7,23 \pm 0,61$ & $0,62 \pm 0,85$ & $80,39 \pm 0,91$ \\
Guaiúba & 532,75 & $77,64 \pm 0,23$ & $0,82 \pm 0,24$ & $19,48 \pm 0,62$ & $1,21 \pm 0,12$ & $0,86 \pm 0,53$ & $32,49 \pm 1,18$ & $0,71 \pm 0,91$ & $108,88 \pm 0,82$ \\
\hline
\end{tabular}

Tabela 2. Composição de ácidos graxos saturados do músculo das espécies de pescado mais produzidas no Estado da Bahia (\%).

\begin{tabular}{lccrrr}
\hline \multicolumn{1}{c}{ Ácidos graxos (\%) } & \multicolumn{3}{c}{ Espécies de pescado } \\
\cline { 2 - 6 } & \multicolumn{1}{c}{ Sardinha } & \multicolumn{1}{c}{ Tainha } & \multicolumn{1}{c}{ Camarão } & Ariacó & Guaiúba \\
\hline C14:0 (mirístico) & $5,00 \pm 0,89$ & $5,26 \pm 0,69$ & $1,38 \pm 0,09$ & $1,66 \pm 1,00$ & $0,80 \pm 0,33$ \\
C15:0 (n-pentadecílico) pentadecílico) & $0,94 \pm 0,15$ & $2,92 \pm 2,57$ & $0,92 \pm 0,03$ & $0,48 \pm 0,08$ & $0,51 \pm 0,18$ \\
C16:0 (palmítico) & $24,02 \pm 2,43$ & $23,34 \pm 0,54$ & $15,35 \pm 0,82$ & $20,07 \pm 1,61$ & $17,16 \pm 2,12$ \\
C17:0 (margárico) & $0,28 \pm 0,12$ & $0,83 \pm 0,54$ & $1,91 \pm 0,08$ & $0,95 \pm 0,69$ & $0,99 \pm 0,17$ \\
C18:0 (esteárico) & $9,05 \pm 2,20$ & $5,04 \pm 1,14$ & $10,90 \pm 0,76$ & $9,48 \pm 067$ & $8,90 \pm 0,76$ \\
C19:0 (n-nonadećlico) & $1,42 \pm 0,15$ & $0,99 \pm 0,15$ & $1,73 \pm 0,12$ & $0,91 \pm 0,09$ & $0,15 \pm 0,19$ \\
C20:0 (araquídico) & $0,44 \pm 0,08$ & $0,14 \pm 0,01$ & - & $0,27 \pm 0,10$ & $0,23 \pm 0,06$ \\
ISAT & 40,62 & 38,52 & 32,19 & 33,80 & 28,74 \\
\hline
\end{tabular}


Tabela 3. Informação nutricional com base em uma porção de 60 gramas do músculo do pescado. Valor diário de referência com base em uma dieta de 2000 calorias.

\begin{tabular}{|c|c|c|c|c|c|c|c|c|c|c|}
\hline \multirow[b]{2}{*}{ Porção } & \multicolumn{2}{|c|}{ Sardinha } & \multicolumn{2}{|c|}{ Tainha } & \multicolumn{2}{|c|}{ Camarão } & \multicolumn{2}{|c|}{ Ariacó } & \multicolumn{2}{|c|}{ Guaiúba } \\
\hline & $60 \mathrm{~g}$ & $\% \mathrm{VD}$ & $60 \mathrm{~g}$ & $\% \mathrm{VD}$ & $60 \mathrm{~g}$ & $\% \mathrm{VD}$ & $60 \mathrm{~g}$ & $\% \mathrm{VD}$ & $60 \mathrm{~g}$ & $\% \mathrm{VD}$ \\
\hline Valor calórico (kcal) & 60 & 3 & 70 & 3 & 60 & 3 & 60 & 3 & 50 & 2 \\
\hline Carboidratos & 0 & 0 & 0 & 0 & 0 & 0 & 0 & 0 & 0 & 0 \\
\hline Proteínas & 12 & 16 & 12 & 16 & 12 & 16 & 12 & 16 & 12 & 16 \\
\hline Gorduras totais & 1,5 & 3 & 3 & 5 & 1 & 2 & 1 & 2 & 0 & 0 \\
\hline Gordura Saturada & 0 & - & 0 & - & 0 & - & 0 & - & 0 & - \\
\hline Gordura Trans & 0 & - & 0 & - & 0 & - & 0 & - & 0 & - \\
\hline Sódio & 120 & 5 & 80 & 3 & 100 & 4 & 60 & 2 & 75 & 3 \\
\hline
\end{tabular}

\subsection{Resultados dos questionários}

Foram analisadas as respostas referentes aos questionários aplicados a 9 (nove) empresas beneficiadoras de pescado do Estado da Bahia, correspondendo a 45\% do total das beneficiadoras do Estado. As empresas estão situadas nas cidades de Paulo Afonso, Jandaira, Salvador, Itacaré, Ilhéus, Ibirapitanga, Mascote, Camacan, Eunapólis, Porto Seguro, Teixeira de Freitas, Caravelas, Valença.

Das beneficiadoras do Estado, 43\% possuem capacidade mensal de produção compreendida entre 10 e 50 toneladas; $28 \%$ possuem produção acima de 10 toneladas; e 28\%, menos de 10 toneladas. Com relação à capacidade de instalações das indústrias, $43 \%$ possuem instalações de mais de $1000 \mathrm{~m}^{2} ; 43 \%$ possuem $500 \mathrm{~m}^{2}$; e $14 \%$, de 800 a $1000 \mathrm{~m}^{2}$.

A maioria das empresas (71\%) utiliza o pescado capturado no seu processamento e apenas $29 \%$ utilizam pescado originário de cativeiro, sendo que os principais produtos finais são: o pescado congelado inteiro (57\%), seguido de pescado congelado filetado (43\%). O principal destino da produção é a região Nordeste (43\%), seguido do mercado internacional (28\%) e da região Sul (14\%).

Cerca de $80 \%$ das empresas produzem um total de resíduos maior que 1 tonelada/mês. Nas beneficiadoras que possuem programa de aproveitamento de resíduos, esse aproveitamento é destinado à produção de farinha de peixe, isca para siri e adubo. Na maior parcela das empresas, não existe um programa apropriado para o destino dos descartes gerados pelo processamento, sendo estes despejados principalmente em aterros, rio, rede de esgoto e beira da estrada, tornando-se importante geradora de poluição do meio ambiente.

A maior dificuldade relatada pelas empresas é a falta de investimento do governo, seguida da sazonalidade da matériaprima, conservação do pescado e falta de novos recursos pesqueiros. Dessa forma, verifica-se que há uma necessidade de qualificação e investimentos, principalmente relacionada a programas de aproveitamento e de descartes de resíduos.

\section{Conclusões}

Todas as espécies apresentaram alto valor protéico.

O ariacó e a guaiúba foram considerados peixes "magros"; a sardinha e o camarão, classificados como "baixo teor de gordura"; e a tainha como "semigordo". A sardinha apresentou maior teor de ácidos graxos saturados.

De acordo com o valor nutricional e o total de ácidos graxos saturados, a guaiúba e o ariacó foram considerados melhor para o consumo.

A maior parte das empresas utiliza o pescado capturado para o processamento, tendo como principal produto o pescado congelado inteiro, sendo o principal destino da produção a região Nordeste.

As maiorias das empresas não possuem um programa apropriado para destino dos descartes gerados pelo processamento, tornando-se fonte de poluição ao meio ambiente.

Os dados obtidos podem ser importantes para a elaboração de tabelas de balanço nutricional, cálculo de ingestão de nutrientes, contribuir para incentivar agregação de valor desse crescente setor pesqueiro do Estado da Bahia.

\section{Referências bibliográficas}

ACKMAN, R. G. Nutritional composition of fats in seafood. Progress in Food and Nutrition Science, v. 13, p. 161-241, 1989.

ALMEIDA, N. M.; FRANCO, M. R. B. Influência da dieta alimentar na composição de ácidos graxos em pescado: aspectos nutricionais e benefícios à saúde humana. Revista Instituto Adolfo Lutz, v. 65, n. 1, p. 7-14, 2006.

ASSOCIATION OF OFFICIAL ANALYTICAL CHEMISTS - AOAC. Cereal foods. In: Arlington, 1997. chap. 32, p. 7-11.

BAHIA PESCA. Boletim estatístico da pesca marítima e estuarina. Estado da Bahia: [s.n.], 2005.

BADOLATO, E. S. G. et al. Sardinhas em óleo comestível. Parte II. Estudo da interação entre os ácidos graxos do peixe e do óleo de cobertura. Revista do Instituto Adolfo Lutz, v. 54, p. 21-26, 1994a.

BADOLATO, E. S. G. et al. Composição centesimal, de ácidos graxos e valor calórico de cinco espécies de peixes marinhos nas diferentes estações do ano. Revista do Instituto Adolfo Lutz, v. 54, n. 1, p. 27-35, 1994b.

BLIGH, E. G.; DYER, W. J. A rapid method of total lipid extraction and purification. Canadian Journal of Biochemistry and Physiology, v. 37, p. 911-17, 1959.

BRASIL. Agência Nacional de Vigilância Sanitária - ANVISA. Resolução RDC n 360, de 23 de dezembro de 2003. Brasília, 2003. 
CASTRO-GONZALEZ, M. I. Omega - 3 fatty acids: benefits and sources. Interciencia, v. 27, p. 128-136, 2002.

HART, F. L.; FISHER, H. J. Análisis moderno de los alimentos. Zaragoza: Acribia, 1971. cap. 10, p. 249.

GOKÇE, M. A. et al. Seasonal variation in proximate and fatty acid compositions of female common sole (Solea solea). Food Chemistry, v. 88, p. 419-423, 2004.

INSTITUTO BRASILEIRO DO MEIO AMBIENTE E DOS RECURSOS NATURAIS RENOVÁVEIS - IBAMA. Monitoramento da atividade pesqueira no litoral do Brasil - Projeto ESTATPESCA: relatório final. Brasília, 2006. Convênio SEAP/IBAMA/ FROZZE $\mathrm{n}^{\circ} 109 / 2004$.

JOSEPH, J. D.; ACKMAN, R. G. Capillary column gas chromatographic method for analysis of encapsulated fish oils and fish oil ethyl esters: collaborative study. Journal of AOAC International, v. 75, p. 488-506, 1992.

KRAUSSE, N. V. et al. Alimentos, nutrição \& dietoterapia. 9. ed. São Paulo: Roca, 1998. 1179 p.

LIMA, M. F. et al. Omega 3 fatty acid (DHA: 22:6 n-3) and neonatal development: aspects related to its essentiality and supplementation. Nutrire: Revista da Sociedade Brasileira de Alimentação e Nutrição, v. 28, p. 65-77, 2004.

LUZIA, L. A. et al. The influence of season on the lipid profile of five commercially important species of Brazilian fish. Food Chemistry, v. 83, p. 93-97, 2003.

MARTIN, C. A. et al. Ácidos graxos poliinsaturados ômega-3 e ômega-6: importância e ocorrência em alimentos. Revista de Nutrição, v. 19, n. 6, 2006.

MOURA, A. F. P. et al. Caracterização da fração lipídica de amostras comerciais de camarão-rosa. Archivos Latinoamericanos de Nutrición, v. 52, n. 2, supl. 2, p.207-211, 2002.

NUNES, M. L. et al. Composição química de alguns peixes marinhos do Nordeste brasileiro. Arquivos de Ciências do Mar, v. 16, n. 1, p. 23-26, 1976.

OGATA, H. Y. et al. Fatty acid composition of five candidate aquaculture species in Central Philippines. Aquaculture, v. 236, p. 361-375, 2004.

OKADA, T.; MORRISSEY, M. T. Seazonal changes in intrinsic characteristics of Pacific sardine (Sardinocs sagax). Journal of Aquatic Food Product Tecnology, v. 16, n. 1, p. 51-71, 2007.

OGAWA, M.; PERDIGÃO, N. B. Aproveitamento industrial de peixes de pequeno porte. Boletim de Ciência do Mar, v. 33, n. 4, p. 1-11, 1979.
ORGANIZAÇÃO DAS NAÇÕES UNIDAS PARA AGRICULTURA E ALIMENTAÇÃO - FAO. The state of world fisheries and aquaculture 2006. Rome: FAO, 2007. p. 3 e 41. Disponível em: <http://www.fao.org/docrep/009/A0699e/A0699E00. htm\#Contents $>$. Acesso em: 9 de maio de 2008.

OLIVEIRA, G. F. Composição centesimal e perfil de ácidos graxos e aminoácidos dos pescados mais comercializados no estado de Santa Catarina. In: PESSATTI, M. L. (Coord.). Aproveitamento dos subprodutos do pescado. Vale do Itajaí: UNIVALI, 2000.

Disponível em: <http://www.gep.cttmar.inivali.br/sub_pesq/etapa1. html $>$. Acesso em: 3 de maio de 2004.

SANCHEZ-MUNIZ, F. J. et al. Deep-frying of sardines in different culinary fats: changes the fatty acid composition of sardines and frying fats. Journal. Agricuture. Food Chemists, v. 40, p. 2252-2256, 1992.

SCHMIDT, M. A. Gorduras inteligentes. São Paulo: Editora Roca LTDA, 2000. $231 \mathrm{p}$.

ROCHA, Y. R. et al. Aspectos nutritivos de alguns peixes da Amazônia. ACTA Amazônia, v. 14, n. 2, p. 787-794, 1982.

RECKS, M. A.; SEABORN, G. T. Variation in fatty acid composition among nine forage species from a southeastern US estuarine and nearshore coastal ecosystem. Fish Physiology Biochemical, v. 34, n. 3, p. 275-287, 2007.

STANSBY, M. E. Polynsaturates and fat in fish flesh. Journal American Dietetic Associantion, v. 63, p. 625-30, 1973.

VISWANATHAN NAIR, P. G.; GOPAKUMAR, K. Fatty acid compositions of 15 species of fish from tropical waters. Journal of Food Science, v. 43, p. 1162-1164, 1978.

VOGEL, A. I. Análise química quantitativa. 5. ed. Rio de Janeiro: Guanabara Koogan, 1992. p. 367-380.

VON SCHACKY, C. Omega-3 fatty acids and cardiovascular disease: lipid metabolism and therapy. Current Opinion in Clinical Nutrition \& Metabolic Care, v. 10, n. 2, p. 129-135, 2007.

YUSUF, H. K. M.; SHARMIN, R. A.; RAFIQUR, R. Fatty acids of 12 marine fish species of the Bay of Bengal. Journal of Food Composition and Analysis, v. 6, p. 346-353, 1993.

WANG, C. et al. Fatty acids from fish or fish-oil supplements, but not $a$-linolenic acid, benefit cardiovascular disease outcomes in primary - and secondary-prevention studies: a systematic review. American Journal of Clinical Nutrition, v. 84, n. 3, p. 5-17, 2006.

ZLATANOS, S.; LASKARIDIS, K. Seasonal variation in the fatty acid composition of three Mediterranean fish - sardine (Sardina pilchardus), anchovy (Engraulis encrasicholus) and picarel (Spicara smaris). Food Chemistry, v. 103, p. 725-728, 2007. 